\title{
Acanthamoeba Keratitis - A Diagnostic Challenge
}

\author{
NADINE A FYDRYSZEWSKI, PETER H HANNA
}

\begin{abstract}
This is a case study of a 23 year old male diagnosed with Acanthamoeba keratitis. Initial misdiagnosis and inappropriate treatment lead to increased severity of the infection requiring surgical intervention. This case illustrates the complexity of Acanthamoeba keratitis infection, highlighting the diagnostic challenges which often begin with misdiagnosis and treatment, the role of the laboratory in providing confirmatory testing, and the relevance of patient knowledge related to contact lens wear and care.
\end{abstract}

INDEX TERMS: Acanthamoeba, keratitis, eye infection, free-living amoeba

Clin Lab Sci 2011;24(4):202

Nadine A. Fydryszewski, PhD, MLS(ASCP), University of Medicine \& Dentistry of New Jersey,, Newark, NJ

Peter H. Hanna MLS(ASCP),New York Presbyterian Weill Cornell Medical Center, New York, NY 10065

Address for Correspondence: Nadine A. Fydryszewski, PhD, MLS(ASCP), University of Medicine \& Dentistry of New Jersey, 65 Bergen Street- SSB GB 20, Newark, NJ 07101-1709,973-972-5089, fydrysna@umdnj.edu

\section{CASE PRESENTATION}

A 23 year old male presented to his ophthalmologist after experiencing two days of pain and redness of the right eye. Additional signs and symptoms included inflammation and some sensitivity to light. The patient history noted extended wear (30 days) soft contact lenses, use of Renu contact lens solution, and no notation about lens wear or a lens care protocol. After eye examination his condition was diagnosed as bacterial conjunctivitis. No cultures were taken. The patient was placed on tobramycin and dexamethasone, a combination antibiotic and steroid ophthalmic suspension, and advised that frequent application of the eye drops would be beneficial.
After one week of therapy there was no improvement. The patient was in constant pain, radiating to the entire right quadrant of the head, and experienced increased light sensitivity necessitating a return to the ophthalmologist. The clinician stated the patient's "cold" was exacerbating his symptoms. Antibiotic drops were discontinued, and a steroid ophthalmic drop, prednisolone, was prescribed for use three times a day. The patient was advised to continue this regime for two weeks.

During this period the patient had difficulty concentrating, blurred vision, pain, and increased sensitivity to light. The patient decided to seek a second opinion. The second ophthalmologist noted contact lens wear, but asked no questions about lens care protocol. After examination, the ophthalmologist stated this was not a bacterial infection but a viral infection, and prescribed trifluridine, an antiviral drug for topical treatment of epithelial keratitis caused by herpes simplex virus. No eye cultures were taken. After three days, the patient showed no improvement. The patient was in constant pain, and exhibited decreased vision in the right eye $(<$ $50 \%$ ), and severe sensitivity to light. He returned to the ophthalmologist who immediately sent him to a corneal specialist at a major eye center in the area.

After initial examination, the corneal specialist stated that the infection was not bacterial or viral, but appeared to be parasitic. At this point, the patient was questioned about his contact lens wear and hygiene protocol. He indicated use of commercial lens solution, discarded lenses every month, but did not discard the lens case. His routine lens case care protocol was rinsing the lens case with tap water. Ophthalmic examination revealed a prominent white ring around the cornea suggestive of Acanthamoeba sp. infection (Figure 1). Corneal scrapings and the contact lens solution from the storage case were sent to the diagnostic laboratory for culture. Both were positive for growth of Acanthamoeba sp. A new therapeutic regime was prescribed utilizing a combination of polyhexamethylene bigua- 


\section{CLINICAL PRACTICE}

nide (PHMB) ophthalmic drops one drop per hour, opthalmadine, one drop every two hours, and an oral antifungal tablet itraconazole one tablet per day.

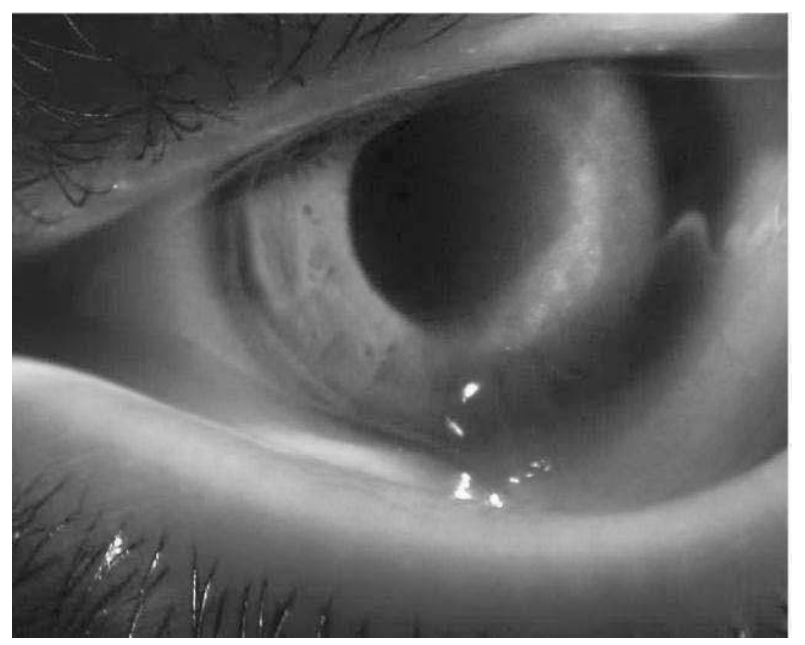

Figure 1. Patient's eye: white ring around cornea. (Courtesey of J. Seedor, MD)

Topical treatments were initially used in an attempt to cure the infection without surgical intervention. After the course of topical treatment, a corneal biopsy was performed to determine if parasites were still present. The biopsy confirmed the presence of cysts, and no trophozoites were present in the cornea. This indicated that the topical treatment was effective in suppressing the parasite's progression. The presence of cysts further confirmed the risk of re-infection without corrective action. Though not curative in this case, the topical treatment limited the spread of the parasite further into the eye. A large graft corneal transplant was performed to ensure excision of the largest possible perimeter where cysts remained. The corneal transplant appeared successful, and two follow-up suture procedures were performed.

The patient was monitored on a weekly basis for the first 30 days post corneal transplant. During this period the patient's vision improved to 60/40, and he received prednisolone ophthalmic drops for inflammation and as an immunosuppressant to avoid graft rejection. Vision continued to improve until the seventh month post corneal transplant. At this point, the patient developed an aggressive cataract as a result of the severe trauma to the eye over the course of the infection. The common protocol to correct the effect of the cataract is to surgically remove the natural lens of the eye and impant a clear artificial lens. In this particular case, the physician chose to use a corrective lens, one with magnification, in order to compensate for some of the vision loss. The corrective cataract lens was surgically implanted, and significantly improved the patient's vision to 20/30. Due to the nature and size of the corneal graft, a high degree of astigmatism was present in the right eye, which was corrected by the use of a precisely fitted contact lens. Eight months from the onset of symptoms the patient's vision was corrected to 20/20.

\section{Acanthamoeba}

Acanthamoeba spp. are small, free-living amoebae. Their phylogenic classification is a Genus within the Kingdom Protista, Class Lobosea, Family Acanthamoebidae, and several species have been identified as agents of human infection. ${ }^{1}$ There are more than 20 species found in the environment, and $A$. polyphaga, and $A$. castellanii are most often associated with human infection. ${ }^{2}$ Acanthamoeba spp. are ubiquitous in nature. They are found in almost all aquatic environments and soil and have been documented in chlorinated swimming pools, tap water, water storage tanks, anaerobic materials such as sludge, feces, many types of soil, and other habitats with low oxygen content. ${ }^{3}$ Acanthamoeba spp. have been associated with human infections of the central nervous system, keratitis, cutaneous lesions and sinusitis and pulmonary infections.

\section{Chronic Granulomatous Amebic Encephalitis}

Acanthamoeba infection of the central nervous system causes chronic granulomatous amebic encephalitis (GAE), and is usually associated with patients having a pre-existing condition such as AIDS, or other chronic illnesses which contribute to an immunocompromised state. ${ }^{4,5,6}$ A chronic infection, GAE lasts more than one week and sometimes even months after the onset of neurological symptoms. Most patients infected with Acanthamoeba of the brain have lowered host resistance. Predisposing factors include diabetes, alcoholism, steroid therapy, drug abuse, and immunocompromised individuals. The transmission is via inhalation of cysts. Excystation occurs quickly, and the trophozoites penetrate the nervous plexus, cross the cribiform plate and enter the brain. The cysts are the infective stage, and characteristics that facilitate infection are viability in a wide temperature range $\left(-20^{\circ} \mathrm{C}\right.$ to $56^{\circ} \mathrm{C}$ ), highly resistant to extreme temperature changes, 


\section{CLINICAL PRACTICE}

disinfection and desiccation. All species that are pathogenic will grow at $37^{\circ} \mathrm{C} .^{3}$

A. culbertsoni is the species most commonly associated with GAE. It leads to meningeal irritation, coma, and death. Clinical symptoms include headaches, seizures, stiff neck, nausea, and vomiting. Granulomatous lesions of the brain develop and may contain the Acanthaomeba trophozoites. In rare cases the trophozoites may invade other areas such as kidney, pancreas, prostate and uterus where lesions will also develop. ${ }^{3}$

\section{Acanthamoeba Keratitis}

A case of ulcerative keratitis was reported as early as 1974. ${ }^{7,8}$ Clinical symptoms resemble corneal herpes simplex virus (HSV) infection, and many cases have been initially misdiagnosed as HSV. A non-healing corneal ulcer is one clinical clue that is associated with parasitic keratitis, and should raise clinical suspicion of a possible Acanthamoeba sp. ocular infection. ${ }^{3}$

Acanthamoeba keratitis (AK) has been increasingly seen in patients wearing contact lenses, predominately daily wear, and extended wear soft lenses. ${ }^{9,10,11}$ Contact lenses can become contaminated with the organism when exposed to non-sterile conditions such as tap or distilled water, dirty hands, saliva, solutions and storage cases that are contaminated. ${ }^{12,13}$ Early cases were traced to saline solutions that were "home-made" using salt tablets and tap water, and poor lens and lens case hygiene. ${ }^{14}$ Another practice that can introduce the parasite to the ocular region is swimming or use of a hot tub while wearing contact lenses. ${ }^{3}$

Contact lens wear increases the ability of the protozoan to penetrate the cornea. The parasites may decrease the corneal integrity, and thus provide a foothold for penetration into the cornea causing an ulcerative lesion. ${ }^{15}$ Clinical symptoms include severe ocular pain which increases with exposure to bright light, vision problems and a prominent white ring around the cornea accompanied by recurrent corneal epithelial breakdown. ${ }^{15}$ Cysts are very difficult to eradicate once established in the cornea where they can reside indefinitely, and perforation of the cornea can result with loss of vision. ${ }^{15}$ Most patients with Acanthamoeba keratitis do not exhibit other systemic symptoms of Acanthamoeba infection. However, there have been cases reported where Acanthamoeba keratitis occurred just prior to, during, or after the development of GAE. ${ }^{16}$

\section{LABORATORY DIAGNOSIS}

Direct microscopic examination, culture, and molecular diagnostic tests are options recommended to confirm the clinical diagnosis. Corneal scrapings and biopsy are the optimal specimens for laboratory diagnostic tests. A microscopic wet mount direct examination of the specimen is recommended using a warmed slide $\left(35^{\circ} \mathrm{C}\right)$. Parasites will exhibit directional movement, but this is not always evident in a wet prep. Acanthamoeba trophozoites range from 15-45 $\mu \mathrm{m}$ with an average of $30 \mu \mathrm{m}$, and have distinctive spiny or thorn-like projections called acanthopodia (Figure 2). There is one nucleus with a large blot-like karyosome and no peripheral chromatin.

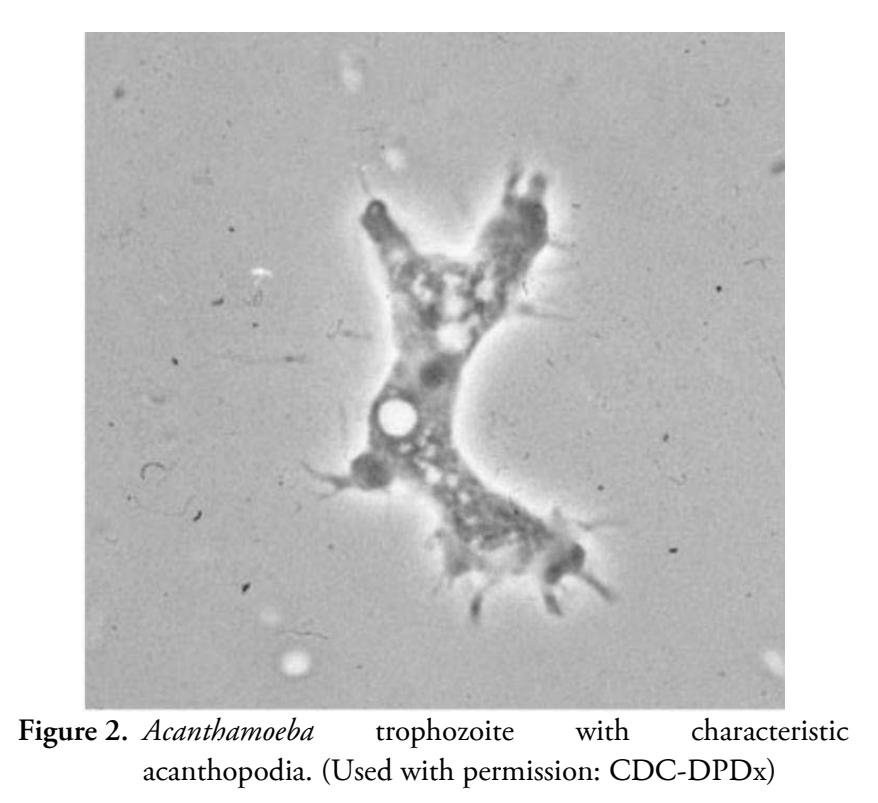

Isolation of the pathogen can be achieved using a nonnutrient agar plate seeded with E.coli, or other gramnegative organisms such as Enterobacter aerogenes, which is recommended when using Culbertson's Medium for Acanthameoba. Other medium recommended for axenic culture are Proteose Peptone-yeast extract-glucose medium, and Trypticase soy broth medium. ${ }^{3}$

The media is inoculated with the specimen suspended in a small amount of Page's ameba saline. ${ }^{3}$ For corneal specimens the recommended incubation temperature is $30{ }^{\circ} \mathrm{C}$, and plates are examined daily for 7 days. ${ }^{3,17,18}$ Agar plate cultures of the organism can yield the cyst form as well as the trophozoite. Cysts typically are 


\section{CLINICAL PRACTICE}

round with a single nucleus and large karyosome. They are double-walled with an inner polyhedral cyst wall and a wrinkled outer wall. Tracks on the agar plate formed by the directional motility of the trophozoite can be observed (Figure 3). Cases have been reported with negative corneal scraping culture but positive culture of contact lens cases. A positive culture of the case may suggest Acanthamoeba infection, but does not confirm Acanthamoeba infection. ${ }^{19,20,21}$

Acanthamoeba, as well as other free-living amoeba, will grow very well in various mammalian cell culture lines. The shell vial technique used in viral isolation provides a good environment, and the cytopathic effect (CPE) observed is similar to what is produced by viruses. ${ }^{3}$ One report of an Acanthamoeba infection of the eye that lead to meningoencephalitis states that the infection was misdiagnosed because of the typical viral CPE observed in cell culture. ${ }^{8}$

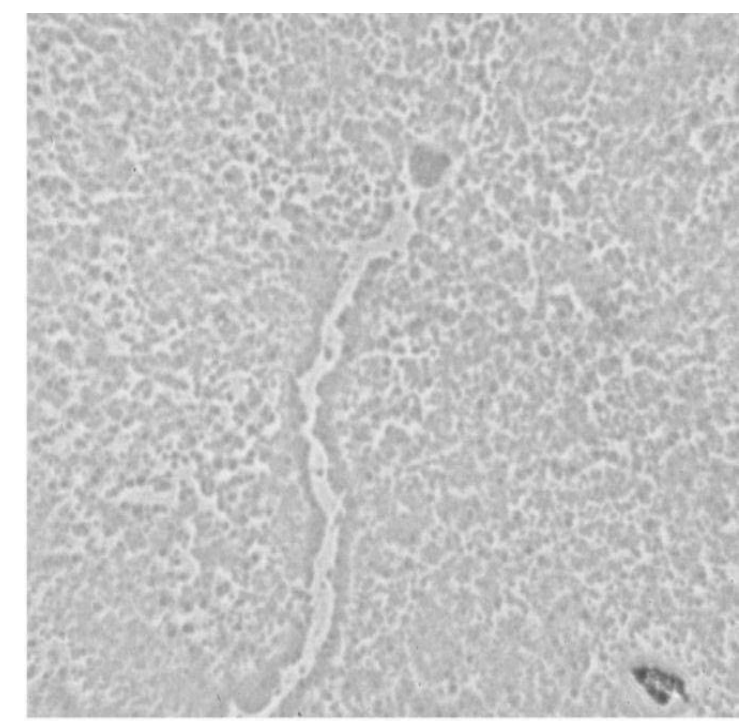

Figure 3. Tracks on agar plate caused by Acanthamoeba troph. 40X (Courtesey of K. VanHorn, PhD)

Various staining methods have been recommended for corneal scrapings and cytological specimens of corneal tissue. Hemacolor stain (Harleco, a division of EM Industries, Inc) is useful for permanently stained smears of corneal scrapings, and fluorescent stains such as calcofluor white and acridine orange can be used to stain cytological specimens. Giemsa stain also works well for corneal scrapings. Other types of histological stains include Periodic Acid Shiff, Gomori's methenamine silver, or trichome..$^{22,23}$
Direct fluorescent antibody (DFA) stains are used in referral and research laboratories as an aid for species differentiation, but these are not commercially available. Molecular detection methods including PCR and fluorescence in situ hybridization have been reported with varying success. ${ }^{24,25,26} \mathrm{PCR}$ is also used by referral and research laboratories to identify free-living ameba to the species level. Several PCR detection methods have been reported in the literature as being successful, but these are not commercially available. A triplex PCR assay that can differentiate Acanthamoeba from the other free-living amoeba associated with human infection, namely Naegleria and Balamuthia, utilizes a design to detect a wide range of Acanthamoeba genotypes. This is in contrast to other assays which detect only one genotype. ${ }^{27,28}$

\section{DISCUSSION/CONCLUSION}

In the case presented, several factors must be considered related to the patient, the clinician, and the laboratorian. Retrospective discussion with the patient revealed that the patient was not aware of the proper protocol for lens care. His failure to follow the proper protocol for lens wear, cleaning, storage and general care played a significant role in the onset of infection. The patient, though using proper cleaning protocol and discarding lenses every month, was not aware of the importance of cleaning the lens case or discarding the lens case monthly. Rinsing the case with tap water, a natural reservoir for this free-living amoeba, as his routine hygiene protocol probably introduced the parasite into the lens case environment. Once in the case, even with appropriate commercially prepared lens solution being used to store lenses, the parasites could adhere to the lens and thus could be introduced into the eye upon daily lens insertion.

Ocular infections with Acanthamoeba spp are often misdiagnosed, as occurred in this case. Early manifestations resemble those of infections with herpes simplex virus, bacterial infections such as Pseudomonas sp. or fungal infections, which contributes to misdiagnosis and delay in appropriate treatment. Topical steroid treatments can exacerbate pathogenicity, so treatment of inflammation with ophthalmic steriods should be. accompanied by adequate amebicidal therapy. ${ }^{29}$ There is evidence that topical steroid treatment might mask the radial keratoneuritis and pseudodendritic lesions that are characteristic in early 


\section{CLINICAL PRACTICE}

stage Acanthamoeba keratitis. ${ }^{30}$ In this case, initial treatment of the infection involved aggressive use of ophthalmic steroid drops which may have contributed to exacerbating the infection and masking the characteristics symptoms.

National and international outbreaks of Acanthamoeba keratitis have been reported in the literature. ${ }^{9,31,32,33,34}$ An increasing number of cases have been reported in the United States between 2004 - 2007. Investigation of cases revealed a commonality of using Complete Moisture Plus (CMP) solution (Advanced Medical Optics, Santa Ana, CA, USA). ${ }^{32}$ Though the investigation did not find the solution contaminated with Acanthamoeba, it was hypothesized that the antiAcanthamoeba efficacy of the solution was, in some way, compromised. ${ }^{32}$

It has been noted that the Acanthamoeba keratitis outbreak of 2004-2007 was similar to a Fusarium keratitis outbreak in 2004-2006 involving CMP solution and $\mathrm{ReNu}$ with MoistureLoc (Bausch \& Lomb, Rochester, NY, USA). ${ }^{32}$ Researchers at Wright State University Boonshoft School of Medicine, Dayton, Ohio, USA conducted experiments with several contact lens solutions including Renu with MoistureLoc. Experiments demonstrated that Renu lost fungistatic activity at a higher rate than other solutions when exposed to prolong periods of increased temperature. $^{35}$ It was suggested that improper temperature control may have contributed to the Fusarium keratitis outbreak. ${ }^{35}$ These findings align with the Food and Drug Administration inspection of the Bausch \& Lomb Greenville facility which manufactured Renu. The FDA cited the facility for inadequacies in temperature control during production, storage, and transport of its products in and beyond the plant. ${ }^{36}$

Clinicians need to consider Acanthamoeba sp. as a possible infectious cause in patients who wear contact lenses. The patient history, with specific questions related to contact lens wear and care protocol, can contribute to the consideration of Acanthamoeba as a possible infectious agent. In this case study, initial patient history noted only that the patient wore contact lenses. No questions were asked about lens care, and the reports from the first two physicians contained no specifics about the patient's lens care protocol. Contact lens wearers need to understand the importance of appropriate lens wear and care protocol in minimizing the chance of lens and lens case contamination with Acanthamoeba. Though the intent is not to scare the patient, knowledge is the most important strategy in preventing infection.

Some laboratorians may not be aware of the appropriate identification procedures for the free living amoeba, such as wet prep, permanent stain and culture, and the possibility of free-living amoeba as parasitic agents of disease. Awareness, knowledge and identification protocols for these parasites can increase a medical laboratory scientist's effectiveness in consulting with clinicians on the potential of free-living amoeba such as Acanthamoeba spp. as parasitic agents of infection. As with all laboratory diagnostic tests, and particularly in microbiology, communication is essential. Patient history can be a vital component for the medical laboratory scientist as a guide to organism identification. Pertinent patient history can aid the laboratorian in the decision-making process related to diagnostic test selection, and potentially infective agents that may be unique or not commonly encountered.

ACKNOWLEDGEMENTS: The authors would like to acknowledge Dr. John Seedor for Figure 1 and Dr. Kenneth G. Van Horn for Figure 3.

\section{REFERENCES:}

1. Rogerson A, Patterson DJ. The naked ramicristate amoebae (Gymnamoebae). In: Lee JJ, Leedale GF, Bradbury P, editors. The illustrated guide to the protozoa. Kansas: Allen Press, Inc.; 2000.

2. Marciano-Cabral F, Guy Cabral G. Acanthamoeba spp. as agents of disease in humans. Clinical Microbiology Reviews. 2003 April;16(2):273-307.

3. Visvesvara GS. Pathogenic and opportunistic free-living amebae. In: Murray PR, Baron EJ, Jorgensen JH, Landry ML, Pfaller MA, editors. Manual of clinical microbiology $9^{\text {th }}$ ed. Washington DC: ASM Press; 2007.

4. Di Gregorio C, Rivasi F, Mongiardo N, De Rienzo B, Wallace S, Visvesvara GS. Acanthamoeba meningoencephalitis in a patient with acquired immunodeficiency syndrome. Archives of Pathology \& Laboratory Medicine.1992;116(12);1363-5.

5. Reed SL. Amebiasis and infections with free-living amebas. In: Kasper DL, Fauci AS, editors. Harrison's Infectious Diseases. New York: McGraw Hill;2010.

6. Dunand VA, Hammer S M, Rossi R, Poulin M, Albrecht MA, Doweiko JP, et al. Parasitic sinusitis and otitis in patients infected with human immunodeficiency virus: report of five cases and review. Clin Infect Dis. 1997;25:267-72.

7. Nagington JP, Watson G, Playfair TJ, McGill J, Jones BR, Steele AD. Amoebic infection of the eye. The Lancet II. 1974; $1537-40$. 


\section{CLINICAL PRACTICE}

8. Jones BR, Visvesvara GS, Robinson NM. Acanthamoeba polyphaga keratitis and acanthamoeba uveitis associated with fatal meningoencephalitis. Trans. Ophthalmol. Soc. U. K. 1975;95:221-32.

9. Thebpatiphat N, Hammersmith KM, Rocha FN, Rapuano CJ, Ayres BD, Laibson PR, et al. Acanthamoeba keratitis: a parasite on the rise. Cornea.2007;Jul;26(6):701-6.

10. Mah-Sadorra JH, Yavuz S, Gulderen A, Najjar D, Laibson PR, RapuanoCJ, et al. Trends in contact lens-related corneal ulcers. Cornea, 2005 Jan;24(1):51-8.

11. Stehr-Green JK, Bailey TM, Visvesvara GS. The epidemiology of acanthamoeba keratitis in the united states. Am J Ophthalmol. 1989 Apr 15;107(4):331-6.

12. Doren GS, Cohen EJ, Higgins SE, Udell IJ, Eagle RC, Arentsen JJ, et al. Management of contact lens associated acanthamoeba keratitis. CLAO J. 1991;17:120-5.

13. Lee JS, Hahn TW, Choi SH, Yu HS, Lee JE. Acanthamoeba keratitis related to cosmetic contact lenses. Clinical and Experimental Ophthalmology 2007 Nov;35(8):775-7.

14. Moore MB, McCulley JP. Acanthamoeba keratitis associated with contact lenses: six consecutive cases of successful management. Br J Ophthalmol. 1989;73:271-5.

15. Omana-Molina M, Gonzalez-Robles A, Salazar-Villatoro LI, Cristobal-Ramos AR, González-Lazaro M, Salinas-Moreno E, et al. Acanthamoeba castellanii: morphological analysis of the interaction with human cornea. Experimental Parasitology 2010 Sept;126(1):73-8.

16. Miller, NR, Hoyt, WF. Walsh and Hoyt's Clinical neuroopthalmology. $6^{\text {th }}$ ed. Philadelphia: Lippincott, Williams \& Wilkins; 2005.

17. D'Aversa G, Stern GA, Driebe WT. Diagnosis and successful medical treatment of acanthamoeba keratitis. Arch. Ophthalmol. 1995;113:1120-3

18. John DT, William AP. Markell and Voge's medical parasitology. $9^{\text {th }}$ ed. St. Louis:Saunders; 2006.

19. Armstrong, M. The pathogenesis of human acanthamoeba infection. Infect. Dis. Rev. 2000;2:65-73.

20. Illingworth CD, Cook SD, Karabatsas CH, Easty DL. Acanthamoeba keratitis: risk factors and outcome. Br. J. Ophthalmol. 1995;79:1078-82.

21. Newman WJ, Browne HB, Seal DV. Acanthamoeba as a "transient" in the corneal scrape of a poorly compliant soft contact lens wearer with peripheral keratitis. Eye. 1997; 11:937-9.

22. Wilhelmus KR, Osato MS, Font RL, Robinson NM, Jones DB. Rapid diagnosis of acanthamoeba keratitis using calcofluor white. Arch. Ophthalmol. 1986;104:1309-12.

23. Hahn TW, O'Brien TP, Sah WJ, Kim JH. Acridine orange staining for rapid diagnosis of acanthamoeba keratitis. Jpn J Ophthalmol. 1998;42:108-14.
24. Khan NA, Jarroll EL, Paget TA. Acanthamoeba can be differentiated by the polymerase chain reaction and simple plating assays. Curr Microbiol. 2001;43:204-8.

25. Lehmann O J, Green SM, Morlet N, Kilvington S, Keys MF, Matheson MM, et al. Polymerase chain reaction analysis of corneal epithelial and tear samples in the diagnosis of Acanthamoeba keratitis. Investig Ophthalmol Visual Sci. 1998;39:1261-5.

26. Mathers W D, Nelson SE, Lane JL, Wilson ME, Allen RC, Folberg R. Confirmation of confocal microscopy diagnosis of acanthamoeba keratitis using polymerase chain reaction analysis. Arch Ophthalmol. 2000;118:178-83.

27. Qvarnstrom Y, Visvesvara GS, Sriram R, da Silva AJ. Multiplex real-time PCR assay for simultaneous detection of Acanthamoeba spp., Balamuthia mandrillaris, and Naegleria fowleri. J Clin Microbiol. 2006;44(10):3589-95.

28. Rivière D, Szczebara FM, Berjeaud J-M, Frère J, Héchard Y. Development of a real-time PCR assay for quantification of Acanthamoeba trophozoites and cysts. J Microbiol Meth. 2006;64(1):78-83.

29. McClellan K, Howard K, Niederkorn JY, Alizadeh H. Effect of steroids on Acanthamoeba cysts and trophozoites. Investig Ophthalmol Visual Sci. 2001;42:2885-93.

30. Saski M, Sotozono C, Chihara H, Ueta M, Inatomi T, Yokoi $\mathrm{N}$, et al. The characteristic appearances of early-stage Acanthamoeba keratitis. Journal of Japanese Ophthalmological Society. 2010 Dec:114(12):1030-5.

31. Joslin CE, Tu EY, McMahon TT, Passaro DJ, Stayner LT, Sugar J. Epidemiological characteristics of a Chicago-area Acanthamoeba keratitis outbreak. Am J Ophthalmol. 2006 Aug:142(2):212-7

32. Verani JR, Lorick SA, Yoder JS, Beach MJ, Braden CR, Roberts JM, et al. National outbreak of Acanthamoeba keratitis associated with use of a contact lens solution, United States. Emerg Infect Dis. 2009 Aug; 15(8):1236-42.

33. Lam DS, Houang E, Fan DS, Lyon D, Seal D, Wong E, et al. Incidence and risk factors for microbial keratitis in Hong Kong: comparison with Europe and North America. Eye (Lond). 2002;16:608-18.

34. Por YM, Mehta JS, Chua JL, Koh TH, Khor WB, Fong AC, et al. Acanthamoeba keratitis associated with contact lens wear in Singapore. Am J Ophthalmol. 2009;148:7-12.e2.

35. Bullock JD, Warwar RE, Elder BL, Northern WI. Temperature instability of $\mathrm{ReNu}$ with MoistureLoc: a new theory to explain the worldwide Fusarium keratitis epidemic of 2004-2006. Arch Ophthalmol. 2008;126:1493-8.

36. US Food and Drug Administration. FDA Form-483 [cited 2007 Jul 28]. http://www.fda.gov/downloads/AboutFDA/ CentersOffices/ORA/ORAElectronicReadingRoom/UCM059 206.pdf 